\title{
Aspectos prácticos de la adhesión a dentina
}

\author{
Martín Hernández J*
}

\section{RESUMEN}

Aunque los sistemas adhesivos han mejorado notablemente, los objetivos que persiguen los nuevos adhesivos son los mismos que perseguían en tiempos de Buonocore. La adhesión debe ser duradera y conseguir siempre que sea posible una interfase cerrada con un sellado perfecto. Se analizan los cuatro aspectos principales que describen una correcta adhesión: Acondicionamiento del substrato, el desarrollo de nuevos adhesivos y técnicas, mecanismos de unión (capa híbrida y tags de resina), resina y su polimerización, sobre todo teniendo en cuenta la contracción de polimerización.

Palabras clave: Adhesión, dentina, técnica.

\section{ABSTRACT}

Although, adhesive systems have improve, objectives of new adhesives continue being the same that Buonocore described. Adhesion must be long-lasting and get a closed interface with perfect sealing. We analyse four main principles that describe adhesion: Substrate conditioning, the development of new adhesive systems and techniques, union mechanisms (hybrid layer and resin tags), resin and it's polymerisation, taking special attention to resin shrinkage.

Key words: Adhesion, dentine, technique.

Aceptado para publicación: octubre 2003.

* Prof. Asociado Facultad de Odontología. Universidad de Valencia.

Martín Hernández J. Aspectos prácticos de la adhesión dentina. Av. Odontoestomatol 2004; 20-1: 19-32.

Desde que en 1955 Buonocuore (1) comenzara a utilizar las técnicas de adhesión a los tejidos duros del diente para conseguir la aplicación de materiales estéticos estas han sufrido una importante y evolución.

Esta evolución ha venido impulsada por un gran número de científicos que se han dedicado intensamente al estudio de todos los aspectos de este proceso. A partir de los estudios de la composición y microanatomía de los tejidos duros así como de la fisiología del complejo dentino-pulpar se han podido desarrollar nuevas técnicas y nuevos adhesivos que han ido perfeccionando la unión del material restaurador al diente, A pesar de ello los objetivos de los nuevos adhesivos siguen siendo los mismos que perseguía Buonocuore en los años cincuenta y que podemos resumir fundamentalmente en dos:

1.- Conseguir una unión suficientemente resistente y duradera del material restaurador al diente.

2.- Conseguir una interfase diente restauración cerrada con un sellado correcto de esta interfase.

En lo que respecta a la unión del diente al material restaurador, si atendemos a lo propuesto por Assmussen en 1988 (2) los avances han sido muy significativos, ya que este autor consideraba que una adecuada resistencia para la interfase adherida 
sometida a fuerzas tangenciales una tensión de 10 $12 \mathrm{MPa}$ ya que fuerzas superiores según pueden producir un fallo cohesivo y no adhesivo.

Las fuerzas de adhesión que estamos manejando con los sistemas adhesivos superan con garantías los $20 \mathrm{MPa}$ en dentina, que siempre a sido el sustrato dental de más difícil adhesión, llegando algunas a alcanzar los 43-45 MPa. Parece pues que el primer objetivo está más que superado con los nuevos adhesivos, lo que no parece estar tan clara es la duración de esa unión con el tiempo y es esta inquietud la que está abriendo nuevos campos de investigación en estos temas.

En cuanto al segundo objetivo parece mucho más lejano de alcanzar pués prácticamente en todos los estudios de filtración de un cierto nivel científico que podemos leer, los autores llegan a la conclusión de que independientemente del adhesivo que estén estudiando en cada momento, el anular totalmente la filtración bien sea micro o nanofiltración es hoy por hoy imposible (3-5).

Y es que la evolución de las técnicas adhesivas aunque espectacular en estos años no ha estado exenta de dificultades que podríamos enumerar resumidamente en 4 fundamentales:

a) La superficie a adherir: Los tejidos duros una vez terminada la eliminación de la caries con la ayuda de instrumental rotatorio, y en concreto la superficie dentinaria que es la que nos ocupa en este trabajo, quedan cubiertas de un barrillo que cubre toda la superficie expuesta tapando el acceso del adhesivo tanto a la dentina peritubular como la intratubular y dificultando así de manera importante la adhesión. Es lo que se ha dado en llamar en la literatura el "barrillo dentinario" o "Smear layer".

b) La humedad de la dentina: es otro factor que dificultaba en los primeros años de perfeccionamiento de las técnicas adhesivas las mismas ya que las primeras resinas que se de que se dispuso tenían un marcado carácter hidrofóbico.

c) Naturaleza hidrofóbica de las primeras resinas: Tanto los primeros adhesivos como los composites de entonces e incluso la mayoría de las resinas compuestas actuales se basan en resinas hidrofóbicas altamente incompatibles con la humedad del sustrato dentinario.

d) Contracción de polimerización de las resinas compuestas: este sigue siendo uno de los mayores enemigos de la integridad de nuestras interfases.

A lo largo de la historia de las técnicas adhesivas, los investigadores han ido planteando distintas soluciones a estos cuatro aspectos principales que vamos a esbozar ahora para describir más detenidamente a lo largo de este trabajo:

a) Preparación de la superficie dentinaria y del esmalte para eliminar el "barrillo dentinario".

b) Desarrollo de nuevas técnicas adhesivas que toleran e incluso "aprovechan" la humedad dentinaria.

c) Desarrollo de nuevas resinas hidrofilicas para los adhesivos.

d) Mejoras en las técnicas de aplicación y polimerización de las reinas compuestas destinadas a minimizar el efecto de la contracción.

\section{Preparación del sustrato}

Este supuso el primer gran avance y quizá hasta el día de hoy el más importante en las técnicas de adhesión a dentina. Basta con leer artículo de Dorfer y cols. Para constatar su importancia aún en nuestros días (6). Esta técnica de grabado o preparación de la superficie dental no se perfeccionó hasta que en 1987 Fusayama no describió su técnica de grabado total con ácido ortofosfórico al 37\% (7).

Antes de Fusayama, a los dentistas nos daba pánico tratar la dentina de dienter vitales con ácidos tan agresivos como el ortofosfórico y reservábamos este para el muy mineralizado esmalte, tratando el "barrillo dentinário" con ácidos más débiles que no comprometieran la vitalidad de los odontoblastos y la pulpa subyacentes a nuestra zona de adhesión. Se sabia que si aplicábamos un ácido fuerte a ambas superficies, esmalte y dentina, durante 40 segundos se conseguía una perfecta adhesión a esmalte (que 
siempre ha sido buena) y una mediocre adhesión a dentina ya que la superficie dentinaria quedaba limpia de barrillo dentinario, con la entrada a los túbulos despejada pero la superficie intertubular e intra tubular totalmente lisa. La aplicación de un ácido fuerte en esmalte y uno debil en dentina conseguía la misma perfecta unión a esmalte y una unión mejor a dentina ya que esta se demineralizaba y dejaba al descubierto una densa alfombra de colágeno sin hidrolizar que cubría la dentina intertubular y la dentina de la entrada a los túbulos dentinarios que mejoraba la adhesión. Fusayama consiguió demostrar que se podia grabar con acidos fuertes durante un tiempo de tan solo 15 segundos y conseguir una adecuada unión del adhesivo al esmalte y una superficie idéntica a la de los ácidos débiles en dentina. La reducción del tiempo de grabado es uno de los factores más importantes en una correcta adhesión como se ha demostrado desde entonces hasta trabajos recientes (6).

Una vez que conocemos como debemos preparar el sustrato a adherir vamos a ver de que adhesivos disponemos en nuestros días para conseguir una interfase adecuada.

\section{Adhesivos}

Una definición de adhesivo dental útil para entender de que estamos hablando sería la siguiente: "Material que colocado en capa fina sirve para adherir el material restaurador al diente, tanto a esmalte como a dentina." No obstante esta definición está obsoleta pues hoy en día es imposible pensar en una correcta adhesión utilizando un solo material, es por ello por lo que parece más correcto que hablemos de Sistemas adhesivos.

Un sistema adhesivo es el conjunto de materiales que nos permiten realizar todos los pasos de la adhesión, es decir, nos permiten preparar la superficie dental para mejorar el sustrato para la adhesión , también nos permiten la adhesión química y micromecánica al diente y por último se unen adecuadamente al material restaurador.

La composición de los adhesivos que encontramos a nuestra disposición en el mercado odontológico, es muy variada y difícil de simplificar, si entráramos en composiciones particulares de cada compuesto del mercado este trabajo se haría eterno y probablemente incompleto pues en ocasiones es difícil obtener datos de la composición cualitativa y cuantitativa de estos productos de las casas comerciales. Además existen magníficos artículos en la literatura con listas interminables de los distintos adhesivos, su composición y distintas clasificaciones $(8,9)$. Es por ello que he tratado de resumir y describir la composición tipo de un adhesivo para matizarla después cuando hablemos de las presentaciones que aparecen en el mercado.

Vamos a ver los componentes fundamentales que forman un sistema adhesivo moderno si bien las pequeñas variaciones en composición pueden tener importancia en el resultado final y conviene conocer a fondo el adhesivo que estemos utilizando.

1. Agente grabador: Los más frecuentemente usados son ácidos fuertes (Ortofosfórico al 37\%) con la técnica de grabado total de Fusayama. También se siguen usando en la composición de los imprimadores ácidos débiles (cítrico maleico etc...) y por último nos encontramos con las nuevas resinas acidicas (Phenil-P, MDP) que actúan como grabadores en los modernos adhesivos autograbantes.

2. Resinas hidrofílicas: Estas son las encargadas de conseguir la unión a dentina impregnando la capa híbrida y formando "tags" aprovechando precisamente la humedad de la dentina. Son resinas como PENTA, HEMA , BPDM, TEGDMA , GPDM o 4-META.

3. Resinas hidrofóbicas: Son las primeras que formaron parte de los materiales adhesivos y aunque son poco compatibles con el agua su función en los sistemas adhesivos es doble, por un lado conseguir una buena unión a la resina compuesta que también es hidrofóbica y por otro conseguir que la capa de adhesivo tenga un grosor suficiente para que nuestra interfase dentina resina soporte el estrés a que se va ver sometida ya que suelen ser más densos que las resinas hidrofilicas.

4. Activadores: Son los encargados de desencadenar la reacción en cascada de la polimerización. 
Basicamente nos encontramos con dos, los fotoactivadores que son las camforoquinonas o el PPD y los quimioactivadores como el complejo Aminaperoxido. En algunas ocasiones se encuentran asociados ambos tipos de activadores y estamos entonces ante un adhesivo de fraguado dual.

5. Relleno inorgánico: Este componente no aparece en todos adhesivos pero en los que lo hace pretende reforzar a través del nanorelleno la resina y conseguir así un adhesivo con propiedades mecánicas mejoradas. Con este tipo de adhesivos es más fácil conseguir un adecuado grosor de capa pues son menos fluidos.

6. Disolventes: En la mayoría de los productos que usamos el solvente es un mero vehículo del producto pero en los sistemas adhesivos este es uno de los componentes fundamentales para conseguir una adhesión adecuada ya que es fundamental para conseguir una adecuada capa híbrida. Por otro lado los solventes muy volátiles como la acetona o el etanol pueden tener problemas en su manipulación por que si dejamos abierto el bote de adhesivo se evaporan con facilidad y la proporción resina solvente se altera y con ella las propiedades del producto $(10,11)$. Es por ello que se están desarrollando nuevos adhesivos en botes monodosis. Los solventes que utilizan nuestros adhesivos son agua, etanol y acetona.

7. En cuanto a la clasificación de los adhesivos tenemos que decir que existen infinitas:

- Unas en función de si los adhesivos eliminan o modifican el barrillo dentinario.

- Otras según su agente grabador los clasifican en:

a. No autograbantes

b. Autograbantes

- También se clasifican según el sistema de activadores que tienen en:

a. Fotopolimerizables.

b. Auto o quimiopolimerizables.

c. Duales

- Según su evolución histórica se han clasificado en adhesivos de $1^{\mathrm{a}}, 2^{\mathrm{a}}, 3^{\mathrm{a}}, 4^{\mathrm{a}}, 5^{\mathrm{a}}$ y $6^{\mathrm{a}}$ generación. Esta es la menos científica de las 4 clasificaciones pero quizá la que nos permite entender mejor la infinidad de presentaciones comerciales en que se nos presentan los adhesivos.

Tendremos solo en cuenta los adhesivos más modernos, de la $4^{\mathrm{a}}$ generación en adelante ya que son los primeros que comienzan a estar diseñados para actuar formando capa híbrida, es decir los primeros diseñados para técnicas adhesivas modernas. (Ver figura 1).

Los adhesivos de $4^{\mathrm{a}}$ generación se presentan normalmente en 3 botes, en el primero (grabador) se encuentra el ácido fuerte (ortofosfórico al 37\%), en el segundo bote (primer o acondicionador) se encuentran las resinas hidrofílicas y el fotoactivador. La función de este es impregnar adecuadamente la dentina. En el tercer bote (bonding o adhesivo) tendremos las resinas hidrofóbicas y los fotoactivadores.

Todos estos adhesivos pueden convertirse en adhesivos de fraguado dual si les añadimos en la composición de acondicionador y adhesivo el peroxido y si el sistema trae un cuarto bote con aminas para mezclarlo en el momento de llevarlo a boca. Esto tiene su interés pues los adhesivos quimiopolimerizables suelen ser más compatibles con las resinas de igual fraguado (utilizadas para cementado) que los fotopolimerizables y viceversa.

Los de la $5^{\mathrm{a}}$ generación surgen del afán de las casas comerciales de reducir los tiempos y el número de pasos de la técnica de aplicación y por tanto de simplificar la técnica. Para ello reducen los botes a dos dejando en el primero el ácido grabador y dejando el segundo para una mezcla de acondicionador y adhesivo de la que forman parte tanto la resina hidrofilica como la hidrofóbica el fotoactivador y el peroxido. Como en los de cuarta eneración, para convertirlos en duales hace falta un tercer bote con las aminas (figura 1). Estos adhesivos han dado en llamarse monocomponentes y son los más usados en la actualidad. Si bien su capacidad adhesiva es algo peor que los de la generación anterior, esta sigue siendo buena $(12,13)$. Y se mejora si añadimos más de una capa de adhesivo con nuestra técnica de aplicación (14).

Los de última generación se han dado en llamar autograbantes. Estos solo tienen un bote que cum- 


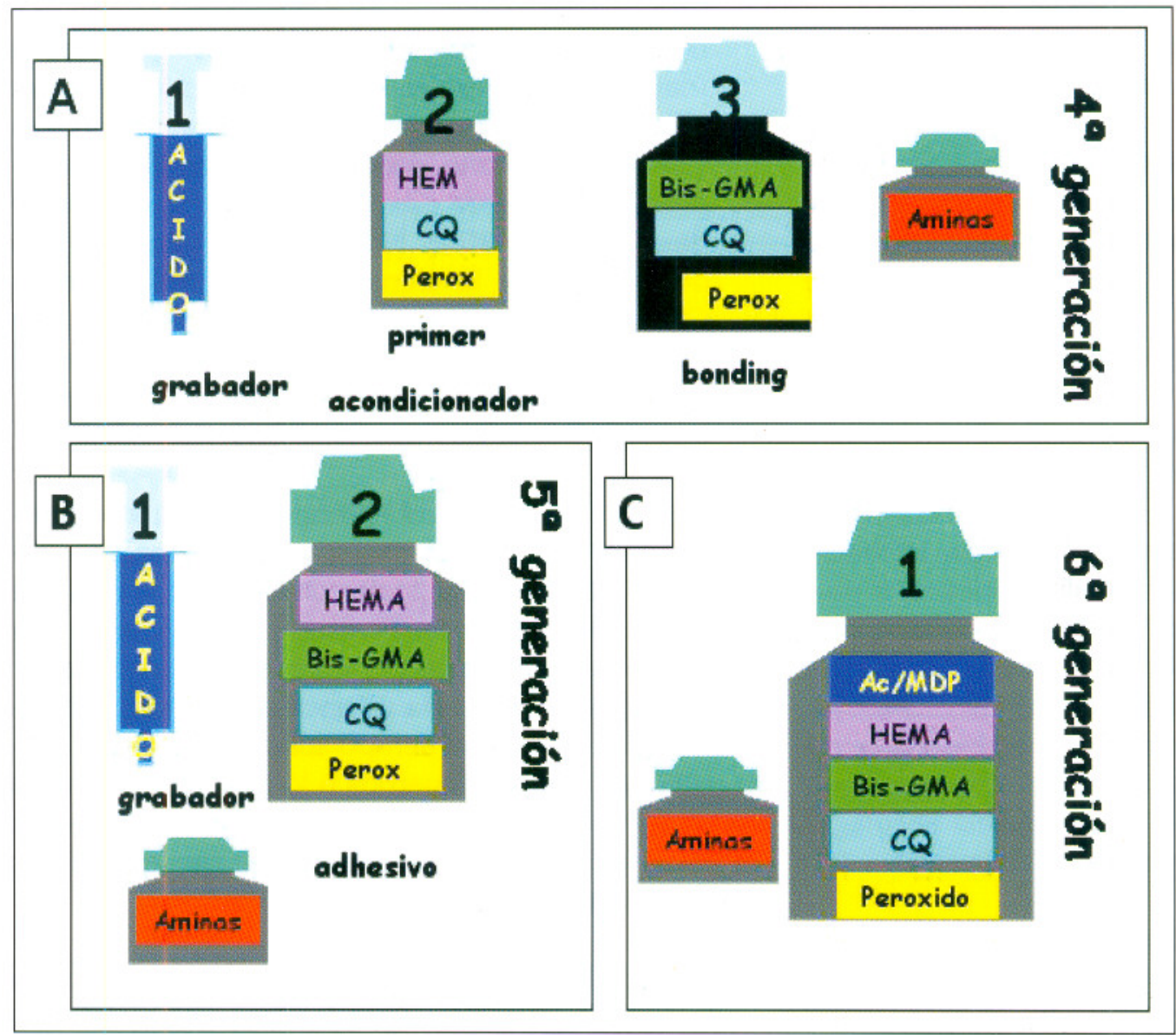

Fig. 1. Equilibrio hídrico en técnica húmeda: A dentina excesivamente seca, colapso de las fibras colágenas. B dentina sobrehumedecída disolución del adhesivo y formación de cuerpos hibroides*. C humedad adecuada correcta impregnación.

estudiemos más detenidamente.

La unión micromecánica se basa en dos estructuras muy importasantes, la "capa híbrida" y los "tags" intratubulares que son dos estructuras cuya formación debemos favorecer con nuestra técnica adhesiva.

La capa híbrida fue descrita como hallazgo microscópico por Nakabayashi en 1982 (15) y confirmado con posterioridad por infinidad de autores $(16,17)$ con posterioridad. Podríamos decir que se forma por la penetración de la resina a través de los nanoespacios que quedan entre las fibras de colágeno desnaturalizadas y expuestas por la acción

ple las tres funciones, la de grabador por que contiene resinas acídicas, la del primer pues contiene la resina hidrofilica y la del bonding por contener la resina hidrofóbica, También contiene los activadores fotoquímicos y puede convertirse en dual añadiéndole las aminas.

Ahora que conocemos los materiales adhesivos y la superficie a adherir, veamos por que mecanismos se unen ambos.

\section{Mecanismos de unión}

Básicamente existen dos mecanismos de unión entre dentina y adhesivo; la unión química que tiene mucha menor importancia cuantitativa y la unión física o micromecánica que parece ser la más importante para mantener la adhesión y que será la que del ácido en la superficie dentinaria y que tras polimerizar, quedan atrapadas en ella. Es por tanto una estructura mixta formada por colágeno de la dentina y resina del adhesivo que encontramos tanto en la superficie de la dentina intertubular como a la entrada de los túbulos dentinarios. La importancia cuantitativa de esta microestructura en la fuerza de adhesión a dentina de los adhesivos dentinarios ha sido sobradamente demostrada siendo más importante que la de los tags (18).

La correcta formación y funcionamiento de esta capa híbrida va a depender de dos factores:

1. Impregnación adecuada de las fibras de colágeno (19).

2. Adecuado grosor de la capa de adhesivo que permita amortiguar en cierto modo las fuerzas que sobre el se van a ejercer (20). 
En la adecuada impregnación del colágeno por la resina intervienen varios factores:

a. Grosor de la capa desmineralizada: Las resinas adhesivas son capaces de infiltrar mejor la dentina totalmente desmineralizada que la dentina parcialmente desmineralizada a menos que la matriz de colágeno se colapse $(17,21,22)$. Sabemos que tiene importancia la longitud de esas fibras de colágeno, es decir el espesor de la "alfombra de colágeno" que tenemos que impregnar. Naka-bayashi (23) piensa que con profundidades de desmineralización de 1-2 mm son suficientes para conseguir cifras de adhesión adecuadas y pueden infiltrarse más fácilmente que desmineralizaciones más profundas. Por otro lado Van Meerbeeck (17) demostró que algunos sistemas adhesivos presentan discrepancia entre la profundidad de desmineralización dentinaria y la de infiltración por la resina lo que no le sorprendió viendo los nano-espacios por los que la resina debe pasar para llegar a infiltrar el colágeno y máxime si ese colágeno está colapsado.

b. Colapso de las fibras de colágeno: Hay muchos autores que han descrito lo que sucede cuando, mediante la técnica adhesiva clásica se secaban profusamente la dentina y el esmalte pera eliminar toda la humedad posible. En el esmalte aparecía una superficie de color blanco tiza que se toma como referencia de un correcto grabado ácido, pero en el a dentina, las fibras de colágeno dejan de "flotar" en el agua y se colapsan formando una capa superficial de colágeno muy compactado que aún dificulta más la difusión de la resina (21, 24-26).

\section{c. Capacidad de difusión intrínseca de los adhesi-} vos: Esta va a depender fundamentalmente del peso molecular de los monómeros del adhesivo y por tanto de su composición química $(15,17,21$, 27). Va a adquirir especial relevancia también la presencia de nanorelleno en la composición de la resina que por un lado mejorará sus propiedades mecánicas pero por otro dificulta la correcta difusión al reducir la fluidez de la resina.

d. Humedad: La naturaleza húmeda del sustrato dentinario de la que ya hemos hablado, ha sido uno de los principales inconvenientes para el desa- rrollo de las nuevas técnicas adhesivas, ya que la mayoría de las resinas que existían tenían carácter hidrofóbico. La búsqueda de una mayor penetración en el sustrato dentinario intentando aprovechar precisamente esa presencia de agua llevó al desarrollo de resinas hidrofóbicas y con ellas a la descripción de la técnica húmeda por Kanca y Gwinnett en 1992 (28-30). Esta técnica trata de aprovechar el agua, como elemento que mantienen las fibras de colágeno erguidas, para coseguir una mejor imbricación entre colágeno y resina.

e. Tiempo: Es este a mi entender uno de los factores más importantes para conseguir una adecuada adhesión y es un factor olvidado en la mayoría de las publicaciones. Para que se produzca una buena impregnación del colágeno y unos "tags" de longitud adecuada es necesario que el adhesivo esté colocado el tiempo suficiente sobre el sustrato sin que lo sequemos o lo polimericemos. La mayoría de fabricantes de adhesivos recomiendan unos 15 segundos para conseguir que estos interactúen adecuadamente con el sustrato.

La técnica húmeda (28-30) parece la más adecuada actualmente para conseguir los mejores resultados en lo que a fuerza adhesiva se refiere, pero esta técnica no está exenta de inconvenientes que derivan fundamentalmente de su complejidad técnica y en concreto de la dificultad que supone mantener el equilibrio hídrico correcto.

La superficie dentinaria en técnica húmeda debe estar ni seca ni mojada sino húmeda y además esta humedad debe estar homogeneamente repartida por toda la superficie y esto es claramente imposible pues, suponiendo que existiera una definición objetiva de lo que es húmedo (que no existe) necesitaríamos cavidades ideales sin rincones para conseguir una distribución uniforme del agua.

El exceso o defecto de humedad tienen gran importancia en el resultado final de nuestra capa híbrida y en la nanofiltración que se produce a través de la misma. En el equilibrio hídrico van a tener gran importancia el aislamiento que realicemos sobre la pieza dental a tratar y el vehículo que presenta el adhesivo para penetrar en el colágeno, es decir el solvente. 


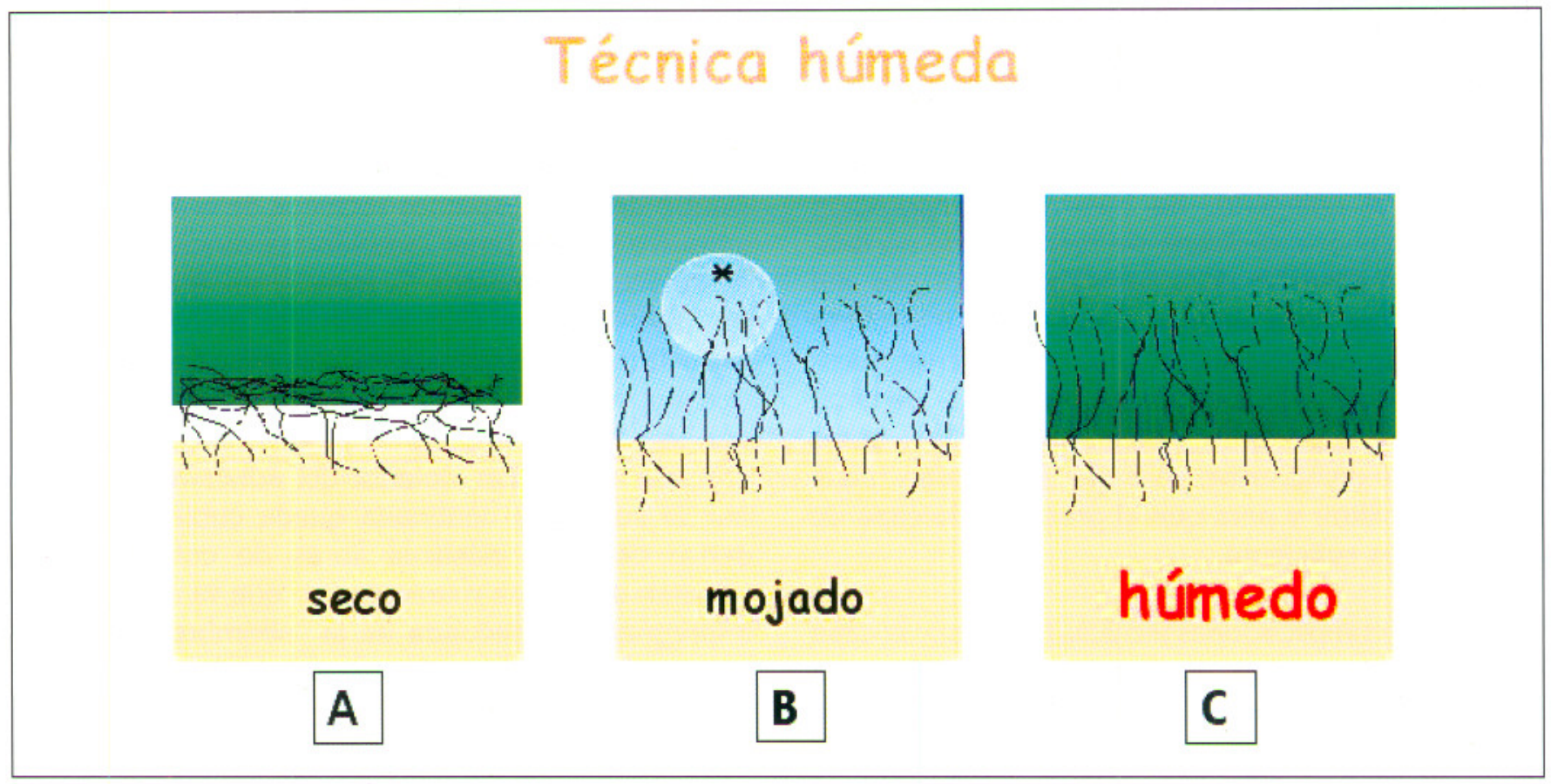

Fig. 2. Composición de los adhesivos: A adhesivos de $4^{\mathrm{a}}$ generación. B adhesivos de $5^{\mathrm{a}}$ generación (monocomponentes). C adhesivos de $6^{\mathrm{a}}$ generación (autograbantes).

En resumen van a influir sobre la calidad de nuestra capa híbrida la técnica, el aislamiento y el solvente del adhesivo.

La humedad que llega a nuestro sustrato dentinario una vez tratado proviene del aporte externo que nosotros hacemos al lavar el ácido y de los túbulos dentinarios que presentan un flujo continuo positivo de fluido dentinario debido a la presión hidrostática positiva de la cámara pulpar.

Si secamos en exceso la superficie dentinaria las fibras colágenas se colapsan y el adhesivo no es capaz de infiltrar hasta la dentina mineralizada (figura 2a), si por el contrario dejamos la superficie dentinaria con exceso de humedad se produce el fenómeno de sobremojado (31) y el adhesivo de disuelve y no adquiere la consistencia adecuada, además se forman en el espesor de la capa híbrida acúmulos de agua en forma de gota que no se infiltran por resina, son los llamados cuerpos hibroides $(31,32)$ (figura $2 b$ ).

En estos fenómenos influye de manera crucial el solvente que presente nuestro adhesivo. Hay tres solventes en los adhesivos comercializados actualmente:

- Acetona: es un solvente que se evapora con mucha facilidad y consigue eliminar por evaporación el exceso de agua si este no es muy importante, es el solvente ideal en condiciones de exceso de agua. Sin embargo es incapaz de reflotar las fibras colágenas colapsadas cuando el sustrato está más seco. Es el peor solvente en situaciones de dentina seca.

- Agua: es lo mismo que encontramos sobre la superficie dentinaria, funciona mal en situaciones de exceso de agua, pero es el mejor en casos de dentina seca ya que es el único que ha demostrado ser capaz de reflotar las fibras de colágeno (33) y por tanto es el único útil en dentina seca.

- Etanol: es un alcohol y por tanto bastante volátil pero no tanto como la acetona, su comportamiento es intermedio entre los dos anteriores.

Por otro lado hay adhesivos que llevan mezclas de dos o tres de estos solventes y por ello cada adhesivo va a tener distinto comportamiento. Otro inconveniente es el almacenamiento de estos materiales que es más delicado cuanto más volátil es el solvente. Si dejamos abierto el bote de adhesivo durante al aplicación del mismo se va evaporando el solvente y la composición del adhesivo va variando desde que estrenamos el bote a las últimas aplicaciones que 
hacemos con el, esto es más importante en los que llevan acetona como solvente (10). Hay que conocer también como debemos eliminar el solvente de la superficie dentinaria para que quede solo la resina infiltrando al colágeno. Se hace por evaporación pero esta hay que realizarla de manera que no desplacemos el adhesivo de la superficie es por ello que los fabricantes recomiendan secar desde una cierta distancia de manera que el chorro de aire de nuestra jeringa no incida directamente sobre la dentina. También es importante aplicar en muchos casos varias capas de adhesivo para que no queden zonas secas sin infiltrar y para que el grosor de la capa de adhesivo sea suficiente.

Todas estas complicaciones técnicas hacen que la capa híbrida pueda ser origen de filtraciones (nanofiltración) que como describió Sano (26) y estudiaron con detalle Tay (34) Armastrong (35) falla a dos niveles cuando sometemos la capa híbrida a pruebas de esfuerzo:

1. En la zona de la capa híbrida más próxima a la dentina, lo que se podría achacare a un defecto en la penetración del adhesivo que deja sin proteger la zona más profunda de las fibras colágenas, también se podría achacar a la degeneración del colágeno pues este tipo de fallo ocurre en las muestras testadas a partir de 180 días.

2. En la zona alta de la capa híbrida, próxima a al resina compuesta. En esta zona es donde se concentran las fibras colágenas y se reduce la proporción de resina adhesiva y aumenta la del colágeno (36).

Todos los errores técnicos de esta técnica húmeda, han sido estudiados ampliamente por Frankenberger (37) en un interesante artículo en el que provoca intencionadamente alteraciones del la cantidad de agua presente en distintos momentos del proceso adhesivo lo que le sirve para justificar la necesidad de un correcto aislamiento para controlar estas situaciones. Otros autores (38) insisten en esta necesidad y lo demuestran contaminando intencionadamente con sangre el proceso de adhesión. Observan que cuando más se reducen las fuerzas de adhesión es si la contaminación con sangre se produce en el momento que las fibras de colágeno ya están expuestas y en segundo lugar tras la aplicación del primer, esto último se soluciona aplicando una nueva capa de primer. No influye la contaminación con sangre tras el grabado ácido si luego se va a proceder a la desproteinización.

Dentro de estos aspectos técnicos de colocación del adhesivo parece tener también relevancia la manera de colocar el adhesivo. Si atendemos a los estudios de Frankenberger (39) el esmalte grabado es una estructura de delicadas espículas muy frágiles y con una importante energía superficial que va a hacer que sea fácil de impregnar y no conviene maltratar con una aplicación violenta del adhesivo, mientras que la superficie dentinaria queda cubierta de una densa capa de elásticas fibras de colágeno que admiten una cierta manipulación que va a mejorar la impregnación adecuada de la "alfombra" colágena como ya defendió Van Meerbeeck (40).

Por otro lado en el mercado existen dos tipos de aplicadores para adhesivos, unos más rígidos con largas cerdas de plástico y otros con un delicado pompon de fibras suaves como los utilizados en endododncia que nos parece más adecuado para movilizar las fibras colágenas de la superficie dentinaria. Resumiendo, debemos usar aplicadores delicados, simplemente acercar el adhesivo al esmalte y restregar de forma suave y repetida la superficie dentinaria para conseguir la mejor impregnación de la superficie dental.

El segundo mecanismo de unión a la dentina en importancia es la formación de tags de resina, es decir prolongaciones resinosas que aprovechan los túbulos dentinarios para conseguir microretención. En al formación de los tags también influyen una serie de factores de muchos de los cuales en cierto modo ya hemos hablado y aquí solo enumeraremos:

a. Hidrofilia de la resina: ya hemos dicho que en los túbulos hay fluido dentinario que por presión hidrostática tiende a salir y mojar la cavidad, con las antiguas resinas hidrofóbicas la formación de taqs era mucho menor que con las actuales hidrofilicas

b. Fluidez de la resina: si la resina es demasiado espesa dificilmente va apenetrar por los túbulos. La fluidez viene determinada por un lado por la estructura química y el peso molecular de la resina y por otro por la presencia o no de nanorelleno inorgánico. 
c. Carga de la resina: es el relleno inorgánico, le confiere mayor viscosidad.

d. Tiempo: factor fundamental y en muchas ocasiones poco valorado desde el punto de vista de la técnica de aplicación. No basta con aplicar la resina hay que darle tiempo a que penetre en los túbulos. La presión asistencial y la necesidad de rentabilizar nuestras consultas muchas veces nos llevan a una dinámica de continua prisa que termina en realizar ciertos procesos como la adhesión dentinaria en el menor tiempo posible. Acabamos por no controlar estrictamente los tiempos que necesita el proceso de adhesión y ese rídiculo ahorro de tiempo, son solo pocos segundos, derivado de no cronometrar estos procesos nos saldrá caro pues tendremos que repetir muchas de nuestras restauraciones lo cual supone una perdida de tiempo mucho mayor. Por otro lado es peor si no tenemos que cambiar la restauración pues nuestro paciente llevará una restauración que no se cae pero está mal adherida al diente con los problemas de filtración marginal que esto acarrea.

e. Tipo de dentina: hasta aquí hemos hablado de la aplicación del adhesivo sobre un sustrato dentinario tipo, es decir ideal, pero ¿Es la dentina igual en todo el diente?. Sabemos que la estructura de la dentina sana es muy distinta si se trata de dentina superficial (alejada de la pulpa) o dentina profunda (próxima a la pulpa), mientras la superficial tiene menor número de túbulos y de diámetro menor, presentando una importante superficie de dentina intertubular, la profunda, presenta un número superior de túbulos de mucho mayor diámetro con lo que la dentina intertubular queda claramente reducida. Y sabemos que estas diferencias influyen en la calidad de nuestra adhesión (41). Pues bien, según estemos trabajando sobre un tipo de sustrato $\mathrm{u}$ otro deberemos tratar de potenciar con nuestra técnica adhesiva el mecanismo adhesivo para el que el sustrato se nos presente más favorable, es decir en la dentina superficial funcionarán mejor adhesivos que formen una inmejorable capa híbrida (por ejemplo los convencionales, no los autogravantes que forman una capa híbrida más débil), mientras que en dentina profunda sin olvidar la capa híbrida pueden funcionar mejor adhesivos que formen buenos tags de resina (por ejemplo los que llevan relleno inorgánico).

Pero no solo hay dentina sana en nuestras restauraciones nos encontramos indefectiblemente con dentina cariada en mayor o menor grado y que tiene también una microestructura muy diferente a la dentina sana y por tanto sus mecanismos de adhesión serán diferentes. Yoshiyama (42) afirma que la adhesión a este tipo de dentina es claramente inferior a la que se produce en dentina sana, que en este tipo de dentina no se forma la capa híbrida tal y como la hemos descrito y que en ambos tipos de dentina, sana y cariada, las fuerzas de adhesión aumentan utilizando técnica húmeda.

También nos encontramos con frecuencia con un tercer tipo de dentina que supone una importante dificultad para la adhesión, la dentina esclerótica. Esta es la típica dentina que nos encontramos en las lesiones abrasivas en filo de cuchillo tan frecuentes en los cuellos dentales. Los autores que han estudiado este tipo de dentina (43-46) llegan a las siguientes conclusiones (figuras 3 y 4 ):

- Que estructuralmente la dentina esclerótica presenta una capa hipermineralizada superficial.

- La capa de dentina hipermineralizada no tiene el mismo espesor en todas las zonas de la lesión siendo más profunda en la parte central de la lesión y más fina en los márgenes de la misma.

- Los túbulos de la dentina esclerótica están obliterados por el depósito de unos tapones de fosfato cálcico.

- Que la dentina esclerótica graba mucho peor que la dentina sana.

- Tras el grabado siempre queda una capa hipermineralizada subsuperficial.

- Tras el grabado ácido los tapones de fosfato cálcico siguen tapando la entrada a los túbulos y sobresalen como pequeñas columnas sobre la superficie grabada.

- La capa de dentina desmineralizada con el grabado 


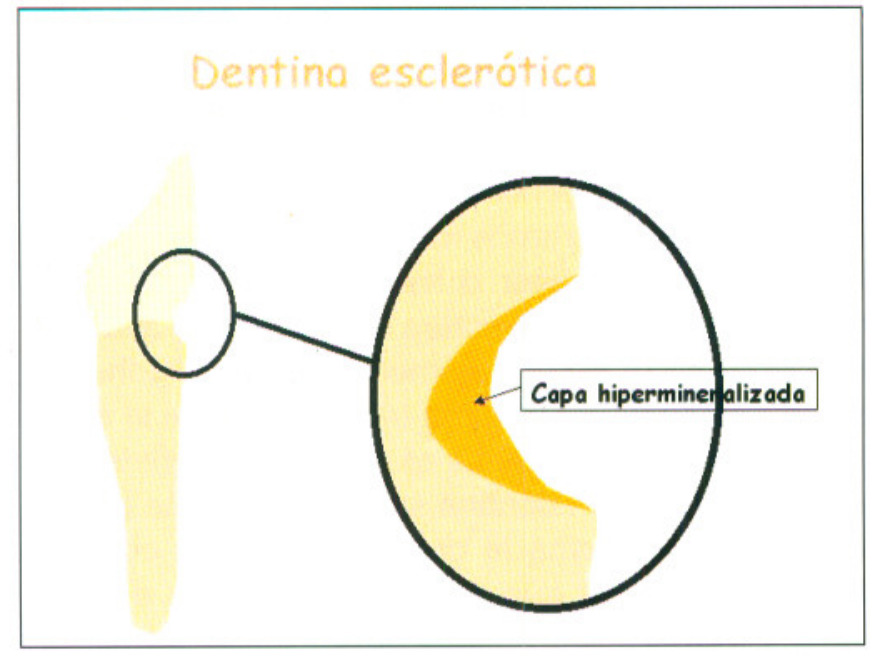

Fig. 3. Lesión esclerótica de la dentina: distinto espesor de la capa hipermineralizada en la lesión.

es pequeña y la capa híbrida que forma es mucho más delgada que la dentina normal.

- Hay una mayor presencia de bacterias que podrían hidrolizar la capa híbrida que en dentina sana.

De todo ello deducen que la adhesión sobre dentina esclerótica es más difícil que sobre dentina sana y que el aumento del tiempo de grabado mejora la adhesión al aumentar el espesor de esa capa híbrida, si bien no llega a alcanzar los niveles de la dentina sana, como tampoco lo consigue la eliminación con la fresa de la capa más superficial de la lesión dadas las diferencias de espesor de la misma dentro de la misma lesion.

Mención merecen también los trabajos de Ogata (47) que estudian la diferente fuerza de adhesión que se consigue sobre la dentina según cortemos los túbulos dentinarios transversal o longitudinalmente. Lo que nos permite afirmar que incluso tratándose de la misma dentina hay notables diferencias de adhesión según la configuración de la cavidad.

Después de tanto estudiar la adhesión a dentina todavía quedan muchas preguntas por responder; ¿Porqué fallan indefectiblemente con el tiempo la inmensa mayoría de nuestras restauraciones? ¿Porqué aparece siempre un cierto grado de filtración cuando estudiamos la adhesión? ¿Tiene importancia clínica este grado de filtración? ¿Es esta la superficie dentinaria ideal para adherir?, ¿Es un elemento fiable para ala adhesión a largo plazo un colá-

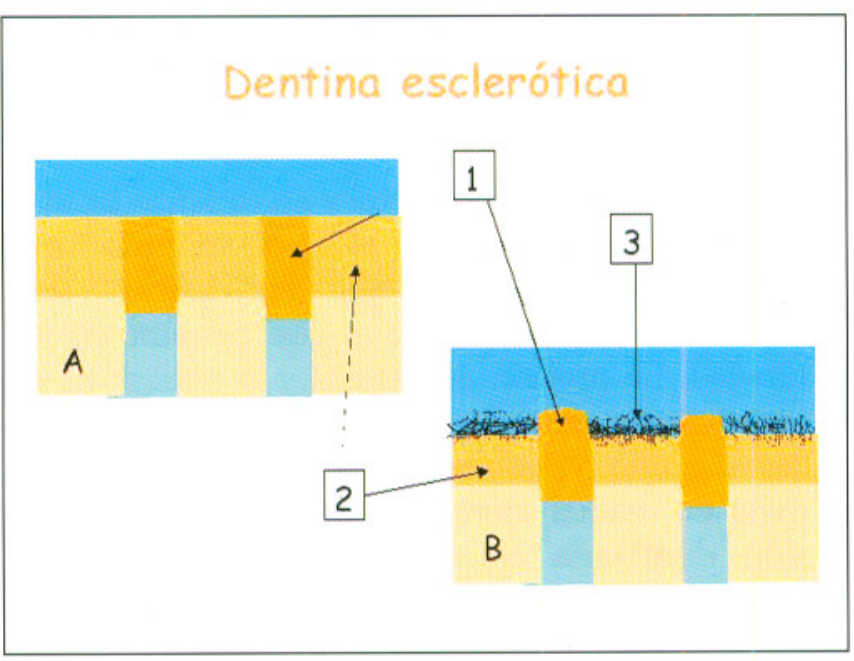

Fig. 4. Dentina esclerótica: microestructura antes $(A)$ y después de grabar (B). 1- Tapones de fosfato calcico. 2- dentina hipermineralizada. 3- Fibras de colágeno, más cortas que en dentina sana.

geno desnaturalizado y difícil de infiltrar por resina?. Intentando dar respuesta a algunas de estas preguntas se están planteando distintos trabajos que nos indican el camino futuro de la adhesión a dentina.

Quiero mencionar aquí los trabajos que están buscando una nueva superficie dentinaria para adherir estudiando la desproteinización de la dentina. Estos parten de la base de que si el colágeno no nos ofrece garantías de durabilidad y además es el principal obstáculo para conseguir una adecuada capa híbrida, ¿̇por qué no lo eliminamos?. Con esta idea se hicieron los primeros trabajos de desproteinización (48) eliminando con la ayuda de hipoclorito sódico las fibras colágenas superficiales de la dentina con ello se conseguía una superficie dentinaria muy atractiva para la adhesión sin elementos orgánicos lábiles y con una superficie mineral más parecida a la que tenemos en el esmalte, que presentaba los túbulos bien despejados y abiertos para recibir a la resina. Cuando se aplicó resina esta penetraba perfectamente en estas estructuras y no solo formaba tags más largos sino que también aparecían infiltradas por resina ramificaciones laterales de los túbulos dentinarios. Sin embargo esta microestructura tan prometedora decepcionó a los investigadores (39) cuando al medir las fuerzas de adhesión que se conseguían a medida que exponían la dentina a mayores tiempos de desproteinización eran cada vez menores (18) mientras que la filtración permanecía en niveles parecidos a los de la adhesión con capa híbrida (49). 
Esto no desanimó a los investigadores que viendo lo que sucedía con los agentes blanqueadores, que reducen temporalmente la capacidad de adhesión al diente, pensaron que en el caso de la desproteinización el fenómeno podía ser parecido y deberse en ambos casos a cambios en el potencial oxidoreductor de la superficie tras la actuación del hipoclorito sódico. Por ello a partir del hallazgo de Itou (50) que veía una mejora en la adhesión sobre superficie dentinaria desmineralizada tratada con ascorbato sódico, Lai y cols (51) han demostrado una mejora en la adhesión a sustrato dentinario desproteinizado con el uso de acido ascórbico que resulta una muy interesante aportación de futuro para estas técnicas.

Parece pues que los grandes avances en adhesión vuelven a pasar por un cambio en el sustrato dental.

Llegados a este punto y volviendo a la técnica técnica adhesiva actual, "técnica húmeda" y antes de dar unas pinceladas a cerca del tercer elemento de nuestra interfase adhesiva, la resina compuesta, es importante reseñar que no se nos debe olvidar polimerizar el adhesivo siempre antes de aplicar la resina compuesta para garantizar la adecuada función del mismo y la adecuada formación y mantenimiento de sus estructuras microrretentivas.

En cuanto a la resina compuesta, solo quiero hacer unas consideraciones generales ya que es otro inmenso campo de estudio que no podríamos desmenuzar en este trabajo si alargarlo excesivamente:

- Las resinas compuestas de que disponemos hoy son hidrofóbicas con lo que obligan a nuestros adhesivos a tener un componente hidrofóbico para unirse a ellas.

- Es recomendable que exista una compatibilidad de fraguado entre resina compuesta y adhesivo, por ello es importante disponer de adhesivos de fraguado dual para permitir mejor unión a los cementos resinosos quimiopolimerizables y a las resinas restauradoras fotopolimerizables.

- Es importante respetar y permitir una adecuada formación de la capa inhibida para conseguir una adecuada union entre adhesivo y resina compues- ta fotopolimerizable y entre las distintas capas de resina compuesta.

- La contracción de fraguado sigue siendo el principal problema para la integridad de las interfases diente-restauración estética y su minimización va a depender de:

1. Desarrollo de nuevas resinas con una contracción mínima.

2. Tener en cuenta el factor de configuración de la cavidad a restaurar y del bloque de resina a polimerizar.

3. Mantener espesores de capa mínimos ala hora de polimerizar a pesar de que las nuevas resinas compuestas puedan polimerizar en espesores mayores ya que la cantidad absoluta de contracción y por tanto el estrés que genera una capa grande es mayor que la de una pequeña aunque porcentualmente sean iguales (52).

4. Uso de capas de materiales interpuestos, resinosos o de otro tipo que permitan con su mayor módulo elástico, amortiguar el estrés de contracción de la resina compuesta en la interfase.

- La forma de polimerizar está adquiriendo mayor relevancia en estos procesos con el paso del tiempo pues parece ser que podemos reducir el tiraje de las resinas compuestas variando la intensidad a lo largo de la polimerización.

\section{Resumen para una adecuada técnica adhesiva:}

Para conseguir una buena técnica adhesiva a dentina hoy en día deberemos tener en cuenta:

1. Realizar un adecuado aislamiento de la pieza.

2. Grabar con acido ortofosfórico al $37 \%$ durante 15 segundos.

3. Lavar adecuadamente la superficie dentinaria y secar hasta dejarla simplemente húmeda. Quiero recalcar en este punto que si no manejamos la técnica húmeda con garantías, recomendamos 
realizar la técnica seca clásica pero utilizando un adhesivo con agua como solvente. Si optamos por la técnica húmeda y prevemos dificultades a la hora de secar la superficie dentinaria reco,mendamos un adhesivo con solvente acetónico.

4. Aplicar el adhesivo con un pincel de punta en bolita de algodón de manera suave sobre el esmalte y más enérgica sobre la dentina intentando impregnar la "alfombra" colágena.

5. Dejar actuar al adhesivo al menos 15 segundos.

6. Eliminar el exceso de solvente evaporándolo suavemente con aire.

7. Aplicar varias capas de adhesivo como suelen recomendar los fabricantes.

8. Polimerizar comenzando con intensidades bajas.

9. Colocar la resina compuesta en finas capas y teniendo en cuenta el factor de configuración de la cavidad a obturar. Si se considera necesario, interponer una capa de material más elástico "amortiguador de tensiones".

10. Polimerizar la resina compuesta comenzando con intensidades de luz bajas.

\section{BIBLIOGRAFÍA}

1. Buonocore MG. "A simple method of increasing the adhesion of acrylic filling materials to enamel surfaces." J Dent Res 1955; 34: 849-53.

2. Assmussen E, Munksgaarde C. "Bonding of restorative resins to dentine: Status of dentine adhesives and impact of cavity design and filling techniques."Int Dent J 1988; 38: 97-104.

3. Del-Nero MO, Escribano N, de la Macorra JC. "Análisis of sealing vs tnsile bond strength of eight adhesive restorative material systems" J Adhes Dent 2000 Summer; 2 (2): 117-27.

4. Bouillaguet S, Duroux B, Ciucchi B, Sano H.
"Ability of adhesive systems to seal dentin surfaces: an in vitro study." J AdhesDent 2000 Autumn; 2 (3): 201-8.

5. Zivkovic S. " Quality assessment of marginal sealing using seven dentin adhesive systems." Quintessence Int 2000 Jun; 31 (6): 423-9.

6. Dorfer CE, Staehle HJ, Wurst MW, Duschner H, Pioch T. " The nanoleakage phenomenom: influence of different dentin bonding agents, termocycling and etching time." Eur J Oral Sci 2000 Aug;108 (4): 346-51.

7. Fusayama T. "Factors and prevention of pulp irritation by adhesive composite resin restorations." Quintessence Int 1997: 18 (9): 633-41.

8. Padrós E, Padrós JL, Manero JM. "Los fastidiosos enigmas de la adhesión dentinaria" Ideas y Trabajos odontoestomatológicos 2000; 1 (1): 837.

9. Van Meerbeeck B, Yoshida Y, Lambrechts P, Vanherle G, Kheogh TP. "Factores que influencian el éxito clínico de la adhesión a dentina y esmalte I“ Maxilaris 1999; 14: 26-36.

10. Gallo JR, Burgess JO, Xu X. " Effect of delayed application on shear bond strength of four fifthgeneration bonding systems" Oper Dent 2001 Jan-Feb; 26 (1): 48-51.

11. Abate PF, Rodriguez VI, Macchi RL. "Evaporation of solvent in one-bottle adhesives." J Dent 2000 Aug; 28 (6): 437-40.

12. Prati C, Chersoni S, Mongiorgi R, Pashley $\mathrm{DH}$. "Resin-infiltrated dentin layer formation of new bonding systems" Oper Dent 1998; 23 (4): 185-94.

13. Wilder AD, Swift EJ, May KN, Waddell SL. "Bond strengths of conventional and simplified bonding systems" Am J Dent 1998; 11 (3): 114-7.

14. Frankenberger R, Perdigao J, Rosa BT, Lopes M. "No-bottle vs multi-bottle dentin adhesives a microtensile bond strength and morphological study" Dent Mater 2001 Sept; 17 (5): 373-80. 
15. Nakabayashi N, Kojima K, Masuhara E. "The promotion of adhesion by the infiltration of monomers into tooth substrates." J Biomed Mater Res 1982; 16: 265-73.

16. Eick JD, Robinson SJ, Byerley TJ et al. "'Scanning transmission electron microscopy / energy-dispersive spectroscopy analysis of the dentin adhesive interface using a labeled 2hydroxyehthylmethacrylate analogue." J Dent Res 1995; 74: 1246-52.

17. Van Meerbeeck B, Inokoshi S, Braem M, Lambrechts P, Vanherle G. "Morphological aspects of the interdiffusion zone observed with different dentin adhesive systems." J Dent Res 1992; 71: 1530-40

18. Perdigao J, Lopes M, Gerardeli S, Lopes GC, García-Godoy F. “ Effect of a sodium hypoclorite gel on dentin Bonding." Dent Mater 2000 Sept; 16 (5): 311-23.

19. Tam LE, Pilliar RM. "The effect of interface stiffness on dentin-composite interfacial fracture resistance." J Dent 2000 Sept; 28 (7): 487-93.

20. Platt JA, Almeida J, Gonzalez-Cabezas C, Rhodes B, Moore BK. " The effect of double adhesive application on the shear bond strength to dentin of compomers using three one-botle adhesive systems." Oper Dent 2001 May -Jun; 26 (3): 313-7.

21. Van Meerbeeck B, Dhem A, Goret-Nicaise $M$, Braem M, Lambrechts P, Vanherle G ." Comparative SEM and TEM examination of the ultraestructure of the resin-dentin interdiffusion zone." J Dent Res 1993; 72: 495-501.

22. Sugizaki J. "The effect of various primers on dentin adhesion of resin composites" Jap J Conserv Dent 199; 34 (1): 228-65.

23. Nakabayashi N. "Resin reinforced dentine due to infiltration of monomers into dentine at the adhesive interface" J Jap Soc Dent Mater Devices 1982; 1: 78-81.
24. Pashley DH, Carvalho RM. "Dentine permeability and dentine adhesion" J Dent 1997; 25 (5): 355-72.

25. Sano H, Yoshiyama M, Ebisu $S$ et al. "Comparative SEM and TEM observations of nanoleakage within the hybrid layer." Oper Dent 1995; 20 (4): 160-7.

26. Sano H, Takatsu T, Ciutcchi B et al. "Nanoleakage: leakage whithin the hirbid layer." Oper Dent 1995; 20 (1): 18-25.

27. Nacabayashi N. "Interpenetration of monomers into dentin and their adhesion" Kokubyo Gakkai Zasshi 1984; 51 (2): 447-54.

28. Kanca J. "Resin bonding to wet substrate I. Bonding to dentin." Quintessence Int 1992; 23: 39-41.

29. Kanca J. "Effect of resin primer solvents and surface wetness on resin composite bond strength to dentin." Am J Dent 1992; 5: 213-5.

30. Gwinnett AJ. "Moist versus dry dentin: its effect on shear bond strength." Am J Dent 1992; 5: 127-9.

31. Tay FR, Gwinnet AJ, Wei SH. "The overwet phenomenon: an optical, micromorphological study of surface moisture in the acid-conditioned, resin-dentin interface." Am J Dent 1996; 9 (1): 43-8

32. Tay FR, Gwinnet AJ, Wei SH. "Micromorphological spectrum from overdying to overwetting acidconditioned dentin in water-free acetone-based, single bottle primer adhesives." Dent Mater 1996; 12 (4): 236-44.

33. Perdigao J, Lambrechts P, Van Meerbeeck B, et al. "The interaction of adhesive systems with human dentin." Am J Dent 1996; 9: 167-73.

34. Tay FR, Carvalho RM, Yiu CK, King NM, Zhang Y, Agee K, Bouillaguet S, Pashley DH. " Mechanical disruption of dentin collagen fibrils during resindentin bond testing." J Adhes Dent 2000; 2(3): 175- 92. 
35. Armstrong SR, Keller JC, Boyer DB. "Mode of failure in the dentin-adhesive resin-resin composite bonded joint as determined by strength-based (muTBS) and fracture-based (CNSB) mechanical testing." Dent Mater 2001; 17 (3): 201-10.

36. Pashley DH, Ciucchi B, Sano H, Horner JA. “ Dentin permeability to adhesive agents." Quintessence Int 1993; 24: 618-31.

37. Frankenberger R, Kramer $N$, Petschelt A. "Technique sensitivity of dentin bonding: effect of application mistakes on bond strength and marginal adaptation." Oper Dent 2000; 25 (4): 32430.

38. Kaneshima T, Yatani H, Kasai T, Watanabe EK, Yamashita A. " The influence of blood contamination on bond strengths between dentin and an adhesive resin cement." Oper Dent 2000; 25 (3): 195-201.

39. Frankenberger R, Kramer N, Petschelt A. "Longterm effect of dentin primers on enamel bond strength and marginal adaptation ." Oper Dent 2000; 25: 11-9.

40. Van Meerbeeck B, Yoshida Y, Lambrechts P, Vanherle G, Kheogh TP. "Factores que influencian el éxito clínico de la adhesión a dentina y esmalte II“ Maxilaris 1999; 15: 20-28.

41. Yoshikawa T, Sano H, Burrow MF, Tagami J, Pashley DH. " Effects of dentin depth and cavity configuration on bond strength." J Dent Res 1999; 78 (4): 898-905.

42. Yoshiyama M, Urayuama A, Kimochi T, Matsuo T, Pashley DH. "Comparison of conventional vs self-etching adhesive bonds to caries affected dentin." Oper Dent 2000; 25 (3): 163-9.

43. Kwong SM, Tay FR, Yip HK, Kei LH, Pashley DH. "An ultrastructural study of the application of dentine adhesives to acid-conditioned sclerotic dentin." J Dent 2000; 28 (7): 515-28.
44. Tay FR, Kwong SM, Itthagarun A, King NM, Yip HK, Moulding KM, Pashley DH. "Bonding of a self-etching primer to non-carious cervical sclerotic dentin: interfacial ultrastructure and microtensile bond strength evaluation." J Adhes Dent 2000; 2 (1): 9-28.

45. Sakoolnamarka R, Burrow MF, Prawer S, Tyas MJ. "Micromorphological investigation of noncarious cervical lesions treated with demineralising agents." J Adhes Dent 2000; 2 (4): 279-87.

46. Marshall GW Jr, Chang YJ, Saeki K, Gansky SA, Marshall SJ. "Citric acid etching of cervical sclerotic dentin lesions: an AFM study." J Biomed Mater Res 2000; 49 (3): 338-44.

47. Ogata M, Okuda M, Nakajima M, Pereira PN, Sano H, Tagami J. "Ifluence of the direction of tubules on bond strength to dentin." Oper Dent 2001; 26 (1): 27-35.

48. Vargas MA, Cobb DS, Armstrong SR. "Resindentin shear bond strength and interfacial ultrastructure with and without hybrid layer. Oper Dent 1997; 22 (4): 159-66.

49. Toledano M, Perdigao J, Osorio R, Osorio E. "Effect of dentin deproteinization on microleakage of class V composite restorations." Oper Dent 2000; 25 (5): 497-504.

50. Itou K, Torii Y, Nishitani Y, Ishikawa K, Suzuki K, Inoue K. "Effect of self-etching primers containing N-acyloyl aspartic acid on dentin adhesion." J Biomed Mater Res 2000; 51 (4): 569-74.

51. Lai SC, Mak YF, Cheung GS, Osorio R, Toledano M, Carvalho RM, Tay FR, Pashley DH. "Reversal of compromised bonding to oxidized etched dentin." J Dent Res 2001; 80 (10): 1919-24.

52. Price RB, Doyle G, Murphy D. "Effects of composite thickness on the shear bond strength to dentin." J Can Dent Assoc 2000; 66 (1): 35-9. 